\title{
Initial Model for USA CoVID-19 Resurgence
}

\author{
Genghmun Eng \\ PhD Physics 1978, University of Illinois at Urbana-Champaign \\ geng001@socal.rr.com
}

September 4, 2020

\begin{abstract}
Early CoVID-19 growth obeys: $N\{\widehat{t}\}=N_{I} \exp \left[+K_{o} \widehat{t}\right]$, with $K_{o}=$ $\left[(\ln 2) /\left(t_{d b l}\right)\right]$, where $t_{d b l}$ is the pandemic growth doubling time. Given $N\{\widehat{t}\}$, the daily number of new CoVID-19 cases is $\rho\{\widehat{t}\}=d N\{\widehat{t}\} / d \widehat{t}$. Implementing society-wide Social Distancing increases the $t_{d b l}$ doubling time, and a linear function of time for $t_{d b l}$ was used in our Initial Model: $N_{o}[t]=\mathbf{1} \exp \left[+K_{A} t /\left(1+\gamma_{o} t\right)\right] \equiv e^{+G_{o}} \exp \left(-Z_{o}[t]\right)$,

to describe these changes, where the $[t]$-axis is time-shifted from the $\{\widehat{t}\}$-axis back to the pandemic start, and $G_{o} \equiv\left[K_{A} / \gamma_{o}\right]$. While this $N_{o}[t]$ successfully modeled the USA CoVID-19 progress from 3/2020 to 6/2020, this equation could not easily model some quickly decreasing $\rho[t]$ cases ("fast pandemic shutoff"), indicating that a second process was involved. This situation was most evident in the initial CoVID-19 data from China, South Korea, and Italy. Modifying $Z_{o}[t]$ to allow exponential cutoffs:

$$
Z_{A}[t] \equiv+\left[G_{o} /\left(1+\gamma_{o} t\right)\right]\left[\exp \left(-\delta_{o} t\right)\right]=Z_{o}[t] \exp \left(-\delta_{o} t\right),
$$$$
N_{A}[t]=e^{+G_{o}} \exp \left(-Z_{A}[t]\right),
$$

resulted in an Enhanced Initial Model (EIM) that significantly improved data fits for these cases.

After 6/2020, many regions of the USA "opened up", loosening their Social Distancing requirements, which led to a sudden USA CoVID-19 Resurgence. Extrapolating the USA $N_{o}[t] 3 / 2020-6 / 2020$ results to $9 / 2020$ as an Initial Model Baseline (IMB), and subtracting this $I M B$ from the newer USA data gives a Resurgence Only function, which is analyzed here. This USA CoVID-19 Resurgence function differs significantly from the $N_{o}[t] I M B$ functional form, but it was well-modeled by the $N_{A}[t]$ fast pandemic shutoff function. These results indicate that: (a) the gradual increase in $t_{d b l}$ doubling time from society-wide shut-downs is likely due to eliminating of a large number of population gathering points that could have enabled CoVID-19 spread; and (b) having a non-zero $\delta_{o}$ fast pandemic shutoff is likely due to more people wearing masks more often [with 12 Figures].
\end{abstract}




\section{Introduction}

The CoVID-19 pandemic started late in 2019, becoming world-wide in early 2020, with CoVID-19 spread evolving differently in various areas. Many publicly available databases were set up to track the disease, to assist epidemiologists, scientists, and policy makers in visualizing CoVID-19 spread. The widely available bing. com $^{\mathbf{1}}$ CoVID-19 database was used here. These databases underpin model projections, allowing quick evaluation of how different inputs affect the predicted outcome. Our goal was to empirically model a wide range of data with a small number of parameters, where different values for these parameters could span the range of observed CoVID-19 evolution among regions.

The $N\{\widehat{t}\}$ number of CoVID-19 cases starts with an exponential growth:

$$
\begin{array}{ll}
N\{\widehat{t}\} \approx N_{I} \exp \left[+K_{o} \widehat{t}\right], & {[1.1 \mathrm{a}]} \\
K_{o} \equiv(\ln 2) / t_{d b l}, & {[1.1 \mathrm{~b}]} \\
d N\{\widehat{t}\} / d \widehat{t}=+K_{o} N\{\hat{t}\}, & {[1.1 \mathrm{c}]}
\end{array}
$$

where $N_{I}$ at time $\widehat{t}=0$ is the number of infected people, $K_{o}$ is a rate constant for how fast an infected person spreads CoVID-19 to others, and $t_{d b l}$ is the pandemic doubling time. This is the basis for a large number of SEIR (Susceptible, Exposed, Infected, and Recovered or Removed) pandemic models, which are often implemented as systems of local differential equations.

Implementing society-wide measures for non-infected people is inherently a non-local process. How it impacts pandemic spread is often not the main focus of SEIR models, which are local. However, when governments mandated Social Distancing, starting with shut-down of large-scale gathering places at some $\widehat{t}=0$ point, the $N\{\hat{t}\}$ response was fairly quick. Within days, the $t_{d b l}$ was empirically observed to gradually lengthen, likely due to these shut-downs preventing a large number of people from gathering together and spreading CoVID-19.

Our prior work ${ }^{2-3}$ showed that an Initial $\mathrm{Model}^{2}$, with a linear function of time for gradual $t_{d b l}$ changes:

$$
\begin{array}{ll}
N\{\widehat{t}\}=N_{I} \exp \left[+K_{o} \widehat{t} /\left(1+\alpha_{S} \widehat{t}\right)\right], & {[1.2 \mathrm{a}]} \\
\underset{t \rightarrow m}{\operatorname{Lim}}[N\{\widehat{t}\}]=N_{I} \exp \left[+K_{o} / \alpha_{S}\right], & {[1.2 \mathrm{~b}]}
\end{array}
$$

successfully fit a lot of early CoVID-19 pandemic data. More importantly, Eq. [1.2b] showed that this Initial Model allows for CoVID-19 pandemic shutoff, prior to infecting the whole population.

An exception was CoVID-19 spread in Italy, having a much faster pandemic shut-off than Eq. [1.2a] predicted. We attributed this to a second CoVID-19 mitigation process that was modeled with a $\delta_{o}$ exponential decay time constant: $U_{A}\{\widehat{t}\} \sim \exp \left(-\delta_{o} \widehat{t}\right)$,

where $\delta_{o}=0$ is the absence of this second process. Including this second process ${ }^{4}$ gave an Enhanced Initial Model (EIM), which then successfully modeled CoVID-19 spread in Italy.

While the Initial Model $^{2-3}$ successfully predicted the USA CoVID-19 evolution from March 2020 through early June 2020, widespread "opening-up" of various gathering places (such as local bars and hair and nail salons) in mid-June 2020 created a large-scale USA CoVID-19 Resurgence. 
A new model for USA CoVID-19 Resurgence is developed here. Our prior (3/2020-6/2020) USA CoVID-19 function was used as an Initial Model Baseline (IMB). This $I M B$ was projected out to $9 / 2020$, and subtracted from all followon USA data, to give a Resurgence Only function. As detailed next, the number of USA CoVID-19 Resurgence cases can substantially exceed the expected preResurgence total. More importantly, this CoVID-19 Resurgence was also found to require a $\delta_{o} \neq 0$ EIM in order to achieve a good data fit.

This $\delta_{o} \neq 0$ result is similar to the prior analysis of CoVID-19 spread in Italy $^{4}$. The fact that the $\delta_{o} \neq 0$ EIM function is needed to model Resurgence, instead of an Eq. [1.2a] IMB-type function, helps to identify the CoVID-19 second process. After the Social Distancing period of 3/20-6/20, new post$6 / 20$ society-wide recommendations or mandates to wear masks were put in place, which likely gives rise to this faster Resurgence pandemic shut-off.

\section{Background}

Let $N\{\hat{t}\}$ model the total number $N_{\text {data }}\{\hat{t}\}$ of CoVID-19 cases in a locality, with $N_{\text {data }}\{\widehat{t}\}$ having end-points $\left\{N_{I}, N_{F}\right\}$. Then $N\{\widehat{t}=0\}=N_{I}$, and:

$$
\begin{aligned}
& N\{\widehat{t}\}=\int_{t^{*}=0}^{t^{*}=\widehat{t}} \rho\left\{t^{*}\right\} d t^{*}+N_{I}, \\
& \rho\{\widehat{t}\}=d N\{\widehat{t}\} / d \widehat{t},
\end{aligned}
$$

where $\rho\{\widehat{t}\}$ is the predicted number of daily new CoVID-19 cases. Early CoVID-19 growth obeys $N\{\widehat{t}\} \approx N_{I} \exp \left[+K_{o} \widehat{t}\right]$, with $K_{o}=\left[(\ln 2) / t_{d b l}\right]$, where $t_{d b l}$ is the pandemic doubling time, but if society-wide Social Distancing starts at $\widehat{t}=0$, then $t_{d b l}$ can lengthen for $\widehat{t}>0$. The prior $\widehat{t}<0$ exponential growth phase, before Social Distancing started, is not applicable for estimating Social Distancing parameters.

Our Initial Model for CoVID-19 spread and $t_{d b l}$ lengthening ${ }^{2-3}$ is given in the above Eqs. [1.2a]-[1.2b]. In Eq. [1.2a], different $N_{I}$ values alter the $\widehat{t}=0$ points. However, all these time axes can be shifted to a new $t=0$ point that estimates the CoVID-19 pandemic start:

$$
\begin{aligned}
& N_{o}[t]=\mathbf{1} \exp \left[+K_{A} t /\left(1+\gamma_{o} t\right)\right] \equiv \exp \left[+G_{o}\right] \exp \left(-Z_{o}[t]\right) \\
& Z_{o}[t]=+\left[G_{o} /\left(1+\gamma_{o} t\right)\right] \\
& G_{o} \equiv\left(K_{A} / \gamma_{o}\right) \\
& \rho_{o}[t] \equiv d N_{o}[t] / d t
\end{aligned}
$$

along with the boundary conditions that $N_{o}\left[t=t_{I}\right] \approx N_{I}$ and $N_{o}\left[t=t_{F}\right] \approx$ $N_{F}$ occur over the $\left(t_{F}-t_{I}\right)$ time interval. The $t_{I}$ value is set by the prior $\left\{K_{o}, \alpha_{S}\right\}$ values. Specifically, at $\widehat{t}=\left[-t_{I}\right]$, Eq. $[1.2 \mathrm{~b}]$ must give:

$$
\mathbf{1} \equiv N_{I} \exp \left\{+K_{o}\left[-t_{I}\right] /\left(1+\alpha_{S}\left[-t_{I}\right]\right)\right\},
$$

which then individually sets $\left\{t_{I}, t_{F}\right\}$ as follows:

$$
\begin{aligned}
& t_{I}=\ln \left(N_{I}\right) /\left[K_{o}+\alpha_{S} \ln \left(N_{I}\right)\right], \\
& t_{F}=\ln \left(N_{I}\right) /\left[K_{o}+\alpha_{S} \ln \left(N_{I}\right)\right]+\left(t_{F}-t_{I}\right),
\end{aligned}
$$

with the $\left\{N_{I}, t_{I}, N_{F}, t_{F}\right\}$ group uniquely determining $\left\{\bar{K}_{A}, \bar{\gamma}_{o}\right\}$ :

$$
\gamma_{o}=\left\{\left[\ln \left(N_{I}\right) / t_{I}\right]-\left[\ln \left(N_{F}\right) / t_{F}\right]\right\} /\left[\ln \left(N_{F}\right)-\ln \left(N_{I}\right)\right],
$$

$$
K_{A}=\left[\left(1 / t_{I}\right)-\left(1 / t_{F}\right)\right] /\left\{\left[1 / \ln \left(N_{I}\right)\right]-\left[1 / \ln \left(N_{F}\right)\right]\right\} \text {, }
$$


which sets the Eq. $[2.2 \mathrm{~b}] Z_{o}[t]$ function. The total number of cases $\left(N_{\max }^{o}\right)$ at the pandemic end, and the long-time tail for $\rho_{o}[t]$ are each given by:

$N_{o}\left[t \rightarrow \infty, Z_{o} \rightarrow 0\right] \approx \mathbf{1} \exp \left[+K_{A} / \gamma_{o}\right]=\exp \left[+G_{o}\right] \equiv N_{\max }^{o}$,

$\rho_{o}[t]=d N_{o}[t] / d t \approx N_{o}[t]\left[G_{o} \gamma_{o} /\left(1+\gamma_{o} t\right)^{2}\right] \rightarrow N_{\max }^{o}\left\{G_{o} /\left[\gamma_{o} t^{2}\right]\right\} \cdot[2.6 \mathrm{~b}]$

The $\left\{0<t<t_{I}\right\}$ period, prior to Social Distancing start, estimates what the pandemic would have looked like, had Social Distancing begun at $t=0$.

Using USA CoVID-19 data from bing. com $^{\mathbf{1}}$ from 3/21/2020 through 6/7/2020, we derived the following Initial Model Baseline (IMB) best fit as shown in Figs. 1-2, using these parameter values:

\begin{tabular}{|c|c|c|c|}
\hline pre- & Initial Model & Date or & \# Cases or \\
\hline Resurgence & Value & Parameter & Value \\
\hline$t=0$ & 0.0 days & $3 / 11 / 2020$ & $N_{00} \approx 1$ \\
\hline data $t_{I}=$ & 9.936 days & $3 / 21 / 2020$ & $N_{I}=23,710$ \\
\hline $\operatorname{data}_{F}=$ & 87.936 days & $6 / 7 / 2020$ & $N_{F}=1,920,628$ \\
\hline calc. $t_{\text {Peak }}=$ & 34.936 days & $4 / 15 / 2020$ & $\left(\begin{array}{c}\rho_{o}\left[t_{\text {Peak }}\right] \approx \\
\mathbf{3 0 . 7 2 7 / \text { day }}\end{array}\right)$ \\
\hline$\left(N_{\max }^{o}\right)=$ & 4,499,494 cases & Max.\# cases & $4,499,494$ \\
\hline calc. $K_{o}=$ & $0.347169 /$ day & calc. $K_{A}=$ & 2.960744 /day \\
\hline calc. $\alpha_{S}=$ & $0.06618 / d a y$ & calc. $\gamma_{o}=$ & $0.193267 /$ day \\
\hline 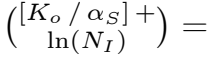 & 15.31948 & $\left(\begin{array}{c}{\left[K_{A} / \gamma_{0}\right]+} \\
\ln \left(N_{00}\right)\end{array}\right)=$ & 15.31945 \\
\hline
\end{tabular}

Shortly after 6/7/2020, many states and cities around the USA "opened up" nearly simultaneously, loosening Social Distancing restrictions. This optimistic action led to a sudden USA CoVID-19 Resurgence.

\section{$3 \quad$ Initial Model for CoVID-19 Resurgence}

To model CoVID-19 Resurgence, the Figs. 1-2 IMB curve values were subtracted from the new USA data totals. When the total number of CoVID-19 Resurgence cases, $\bar{N}_{\text {data }}\{\widehat{t}\}$, showed a trend above the IMB baseline, then $\left\{\bar{N}_{I}, \bar{N}_{F}\right\}$ could be used as the $\bar{N}_{\text {data }}\left\{\widehat{t}^{\prime}\right\}$ data end-points. Let $\bar{N}\left\{\widehat{t}^{\prime}\right\}$ model this CoVID-19 Resurgence data, so that $\bar{N}\{\widehat{t}=0\}=\bar{N}_{I}$. Then:

$$
\begin{aligned}
& \bar{N}\left\{\widehat{t}^{\prime}\right\}=\int_{t^{*}=0}^{t^{*}=\widehat{t}^{\prime}} \bar{\rho}\left\{t^{*}\right\} d t^{*}+\bar{N}_{I}, \\
& \bar{\rho}\left\{\widehat{t^{\prime}}\right\}=d \bar{N}\left\{\widehat{t^{\prime}}\right\} / d \widehat{t}^{\prime},
\end{aligned}
$$

where $\bar{\rho}\left\{\widehat{t}^{\prime}\right\}$ is the predicted number of daily new CoVID-19 Resurgence cases. Early CoVID-19 Resurgence can obey $\bar{N}\left\{\widehat{t}^{\prime}\right\} \approx \bar{N}_{I} \exp \left[+\bar{K}_{o} \widehat{t}^{\prime}\right]$, where $\bar{K}_{o}=\left[(\ln 2) / \bar{t}_{d b l}\right]$ and $\bar{t}_{d b l}$ is the pandemic Resurgence doubling time. Using an Initial Resurgence Model (IRM) that parallels the prior section IMB gives:

$$
\begin{aligned}
& \bar{N}\left\{\widehat{t^{\prime}}\right\}=\bar{N}_{I} \exp \left[+\bar{K}_{o} \widehat{t}^{\prime} /\left(1+\bar{\alpha}_{S} \widehat{t}^{\prime}\right)\right], \\
& \underset{\hat{t}^{\prime} \rightarrow \infty}{\operatorname{Lim}}\left[\bar{N}\left\{\hat{t}^{\prime}\right\}\right]=\bar{N}_{I} \exp \left[+\bar{K}_{o} / \bar{\alpha}_{S}\right] .
\end{aligned}
$$

The best $\left\{\bar{K}_{o}, \bar{\alpha}_{S}\right\}$ are set by minimizing the rms error between the Eq. [3.2a] function and the measured CoVID-19 Resurgence data. In Eq. [3.2a], the $\widehat{t}^{\prime}<0$ data, where $\bar{N}\left\{\widehat{t}^{\prime}\right\}<\bar{N}_{I}$, are not used to estimate the $\left\{\bar{K}_{o}, \bar{\alpha}_{S}\right\}$ pandemic 
Resurgence parameters. As Eq. [3.2b] shows, this IRM allows pandemic shutoff before the CoVID-19 Resurgence infects the whole population. In Eq. [3.2a], different $\bar{N}_{I}$ values alter the $\widehat{t}=0$ points, but all these time axes can be shifted to a new $t^{\prime}=0$ point that estimates the CoVID-19 Resurgence start:

$$
\begin{array}{lr}
\bar{N}_{o}\left[t^{\prime}\right]=1 \exp \left[+\bar{K}_{A} t^{\prime} /\left(1+\bar{\gamma}_{o} t^{\prime}\right)\right] \equiv \exp \left[+\bar{G}_{o}\right] \exp \left(-\bar{Z}_{o}\left[t^{\prime}\right]\right), & {[3.3 \mathrm{a}]} \\
\bar{Z}_{o}\left[t^{\prime}\right]=+\left[\bar{G}_{o} /\left(1+\bar{\gamma}_{o} t^{\prime}\right)\right], & {[3.3 \mathrm{~b}]} \\
\bar{G}_{o} \equiv\left(\bar{K}_{A} / \bar{\gamma}_{o}\right), & {[3.3 \mathrm{c}]} \\
\bar{\rho}_{o}\left[t^{\prime}\right] \equiv d \bar{N}_{o}\left[t^{\prime}\right] / d t^{\prime} . & {[3.3 \mathrm{~d}]}
\end{array}
$$

Since the $\left\{\bar{N}_{I}, \bar{N}_{F}\right\}$ data end points span a time interval of $\left(\bar{t}_{F}-\bar{t}_{I}\right)$, the constraints $\bar{N}_{o}\left[t^{\prime}=\bar{t}_{I}\right]=\bar{N}_{I}$ and $\bar{N}_{o}\left[t^{\prime}=\bar{t}_{F}\right]=\bar{N}_{F}$ determines $\left\{\bar{K}_{A}, \bar{\gamma}_{o}\right\}$ as follows. Using a method similar to Eq. [2.3] and Eqs. [2.4a]-[2.4b], the best fit $\left\{\bar{K}_{o}, \bar{\alpha}_{S}\right\}$ values from Eq. [3.2a] set $\left\{\bar{t}_{I}, \bar{t}_{F}\right\}$ :

$$
\begin{aligned}
& \bar{t}_{I}=\ln \left(\bar{N}_{I}\right) /\left[\bar{K}_{o}+\bar{\alpha}_{S} \ln \left(\bar{N}_{I}\right)\right], \\
& \bar{t}_{F}=\ln \left(\bar{N}_{I}\right) /\left[\bar{K}_{o}+\bar{\alpha}_{S} \ln \left(\bar{N}_{I}\right)\right]+\left(\bar{t}_{F}-\bar{t}_{I}\right),
\end{aligned}
$$

with the $\left\{\bar{N}_{I}, \bar{t}_{I}, \bar{N}_{F}, \bar{t}_{F}\right\}$ group uniquely determining $\left\{\bar{K}_{A}, \bar{\gamma}_{o}\right\}$ :

$$
\begin{aligned}
& \bar{\gamma}_{o}=\left\{\left[\ln \left(\bar{N}_{I}\right) / \bar{t}_{I}\right]-\left[\ln \left(\bar{N}_{F}\right) / \bar{t}_{F}\right]\right\} /\left[\ln \left(\bar{N}_{F}\right)-\ln \left(\bar{N}_{I}\right)\right], \\
& \bar{K}_{A}=\left[\left(1 / \bar{t}_{I}\right)-\left(1 / \bar{t}_{F}\right)\right] /\left\{\left[1 / \ln \left(\bar{N}_{I}\right)\right]-\left[1 / \ln \left(\bar{N}_{F}\right)\right]\right\},
\end{aligned}
$$

which sets the Eq. [3.3b] $\bar{Z}_{o}\left[t^{\prime}\right]$ function. The total number of cases $\left(\bar{N}_{\max }^{o}\right)$ at pandemic end, and the long-term tail for $\bar{\rho}_{o}\left[t^{\prime}\right]$ are each given by:

$$
\begin{gathered}
\bar{N}_{o}\left[t^{\prime} \rightarrow \infty, \bar{Z}_{o} \rightarrow 0\right] \approx 1 \exp \left[+\bar{K}_{A} / \bar{\gamma}_{o}\right]=\exp \left[+\bar{G}_{o}\right] \equiv \bar{N}_{\max }^{o}, \\
\bar{\rho}_{o}\left[t^{\prime}\right]=d \bar{N}_{o}\left[t^{\prime}\right] / d t^{\prime}= \\
\bar{N}_{o}\left[t^{\prime}\right]\left[\bar{G}_{o} \bar{\gamma}_{o} /\left(1+\bar{\gamma}_{o} t^{\prime}\right)^{2}\right] \rightarrow \bar{N}_{\max }^{o}\left[\bar{G}_{o} /\left\{\bar{\gamma}_{o}\left(t^{\prime}\right)^{2}\right\}\right],
\end{gathered}
$$

\begin{tabular}{|c|c|c|c|}
\hline Resurgence & $\begin{array}{c}\text { Initial Model } \\
\text { Value }\end{array}$ & $\begin{array}{c}\text { Date or } \\
\text { Parameter }\end{array}$ & $\begin{array}{c}\text { \# Cases or } \\
\text { Value }\end{array}$ \\
\hline$t^{\prime}=0$ & 0.0 days & $5 / 31 / 2020$ & $\bar{N}_{00} \approx 1$ \\
\hline $\operatorname{data} \bar{t}_{I}=$ & 12.72 days & $6 / 13 / 2020$ & $\bar{N}_{I}=15,650$ \\
\hline data $\bar{t}_{F}=$ & 68.72 days & $8 / 8 / 2020$ & $\bar{N}_{F}=2,213,058$ \\
\hline calc. $\bar{t}_{\text {Peak }}=$ & 66.72 days & $8 / 6 / 2020$ & $\left(\begin{array}{l}\bar{\rho}_{o}\left[{ }_{\bar{t}} \text { Peak }\right] \approx \\
\mathbf{5 4 , 8 6 6 / \text { day }}\end{array}\right)$ \\
\hline$\left(\bar{N}_{\max }^{o}\right)=$ & $15,179,600$ cases & Max.\# cases & $15,179,600$ \\
\hline $\bar{K}_{o}=$ & $0.3158 /$ day & $\bar{K}_{A}=$ & $1.82566 / d a y$ \\
\hline $\bar{\alpha}_{S}=$ & $0.04592 / d a y$ & $\bar{\gamma}_{o}=$ & 0.11041 /day \\
\hline$\left(\begin{array}{c}{\left[\bar{K}_{o} / \bar{\alpha}_{S}\right]+} \\
\ln \left(\bar{N}_{I}\right)\end{array}\right)=$ & 16.5354 & $\left(\begin{array}{c}{\left[\bar{K}_{A} / \bar{\gamma}_{o}\right]+} \\
\ln \left(\bar{N}_{00}\right)\end{array}\right)=$ & 16.5353 \\
\hline
\end{tabular}

showing that $\bar{\rho}_{o}\left[t^{\prime}\right]>0$. Either $\left\{\bar{K}_{A}, \bar{\gamma}_{o}\right\}$ or $\left\{\bar{G}_{o}, \bar{\gamma}_{o}\right\}$ can be used as the primary variables. The IRM analysis results for the USA CoVID-19 Resurgence are shown in Figs. 3-4, using the best-fit $\left\{\bar{G}_{o}, \bar{\gamma}_{o}\right\}$ values. The prior $\{0<$ $\left.t^{\prime}<\bar{t}_{I}\right\}$ period in Fig. 3 estimates what the pandemic would have looked like, if Resurgence Social Distancing had begun at $t^{\prime}=0$. The best fit parameter values for Figs. 3-4 are:

Unfortunately, Fig. 3 shows that the $\bar{\rho}_{o}\left[t^{\prime}\right] I R M$ data fit is not that good. The $\bar{\rho}_{o}\left[t^{\prime}\right]$ function has a long tail, which overestimates $\bar{N}_{\text {max }}^{o}$, as the maximum number of CoVID-19 Resurgence cases at the pandemic end. 


\section{Enhanced Model: USA CoVID-19 Resurgence}

Since the observed $\bar{\rho}_{o}\left[t^{\prime}\right]$ Resurgence data decreases much quicker than the IRM prediction, the USA CoVID-19 Resurgence has a fast pandemic shutoff, which is similar to our prior study ${ }^{\mathbf{3}-\mathbf{4}}$ of Italy CoVID-19 data. That Italy data was most successfully modeled by introducing a second process having an exponential decay in time. Generalizing the IRM model of Eq. [3.3a] similarly gives this Enhanced Initial Model (EIM) for Resurgence, where $\bar{\delta}_{o} \neq 0$ in Eq. [4.1c] characterizes this second process:

$$
\begin{aligned}
& N_{A}\left(t^{\prime}\right)=\mathbf{1} \exp \left[+\bar{G}_{o}\right] \exp \left[-Z_{A}\left(t^{\prime}\right)\right], \\
& Z_{A}\left(t^{\prime}\right)=\bar{Z}_{o}\left[t^{\prime}\right] U_{A}\left(t^{\prime}\right)>0 \\
& U_{A}\left(t^{\prime}\right) \equiv+\exp \left(-\bar{\delta}_{o} t^{\prime}\right) \\
& \bar{Z}_{o}\left[t^{\prime}\right]=+\left[\bar{G}_{o} /\left(1+\bar{\gamma}_{o} t^{\prime}\right)\right] \\
& Z_{A}\left(t^{\prime}=0\right) \equiv+\bar{G}_{o}=\left(\bar{K}_{A} / \bar{\gamma}_{o}\right)
\end{aligned}
$$

The above Eqs. [4.1a]-[4.1e] have these limits:

$$
\begin{aligned}
& Z_{A}\left(t^{\prime} \rightarrow \infty\right) \equiv 0 \\
& N_{A}\left(t^{\prime}=0\right)=\mathbf{1} \\
& U_{A}\left(t^{\prime}=0\right) \equiv+1, \\
& U_{A}\left(t^{\prime} \rightarrow \infty\right) \equiv 0, \\
& N_{A}\left(t^{\prime} \rightarrow \infty\right)=\mathbf{1} \exp \left[+\bar{G}_{o}\right] .
\end{aligned}
$$

For easier data fitting when $\delta_{o} \neq 0$, the Eq. [4.2b] condition that $N_{A}\left(t^{\prime}=\right.$ $0)=1$ can be relaxed. Adjusting $N_{A}\left(t^{\prime}=0\right)$ allows $N_{A}\left(t^{\prime}=\bar{t}_{I}\right)=\bar{N}_{I}$ to be preserved. Then both $\left\{\bar{t}_{I}, \bar{N}_{I}\right\}$ can be treated as model inputs. The $\bar{G}_{o}$ prefactor in Eq. [4.1a] can be modified to give:

$$
N_{A}\left(t^{\prime}\right)=\mathbf{1} \exp \left[+\bar{G}_{o}-\bar{h}_{A}\right] \exp \left[-Z_{A}\left(t^{\prime}\right)\right],
$$

so that $\bar{h}_{A}$ can adjust $N_{A}\left(t^{\prime}=0\right)$, while keeping the same $t^{\prime}=0$ point:

$$
\begin{aligned}
& N_{A}\left(t^{\prime}=0\right)=\mathbf{1} e^{-\bar{h}_{A}}, \\
& N_{A}\left(t^{\prime}=\bar{t}_{I}\right)=\mathbf{1} e^{-\bar{h}_{A}} \exp \left[+\bar{G}_{o}\right] \exp \left[-Z_{A}\left(\bar{t}_{I}\right)\right] \equiv \bar{N}_{I} . \\
& \lim _{t \rightarrow \infty}\left[N_{A}\left(t^{\prime}\right)\right] \equiv \mathbf{1} e^{-\bar{h}_{A}} \exp \left[+\bar{G}_{o}\right]=\mathbf{1} e^{-\bar{h}_{A}} \exp \left[+\bar{K}_{o} / \bar{\gamma}_{o}\right]=N_{A}^{\max } \cdot[4.4 \mathrm{~b}] \\
&
\end{aligned}
$$

Here $N_{A}^{\max }$ is the total number of CoVID-19 Resurgence cases at the pandemic end for this EIM model. Given values for $\left\{\bar{G}_{o}, \bar{\gamma}_{o}, \bar{\delta}_{o}, \bar{t}_{I}, \bar{N}_{I}\right\}$, Eq. [4.4b] uniquely sets $\bar{h}_{A}$ via:

$$
\begin{aligned}
& \bar{h}_{A} \equiv \bar{G}_{o}-Z_{A}\left(\bar{t}_{I}\right)-\ln \left(\bar{N}_{I}\right) . \\
& Z_{A}\left(t_{I}\right) \equiv \bar{Z}_{o}\left(t_{I}\right) U_{A}\left(t_{I}\right)>0 .
\end{aligned}
$$

The $N_{A}\left(t^{\prime}\right)$ of Eq. [4.3] then gives this $\rho_{A}\left(t^{\prime}\right)$ :

$$
\begin{gathered}
\rho_{A}\left(t^{\prime}\right) \equiv \frac{d}{d t^{\prime}} N_{A}\left(t^{\prime}\right)=\mathbf{1} \exp \left[+\bar{G}_{o}\right] \frac{d}{d t} \exp \left[-Z_{A}\left(t^{\prime}\right)\right]= \\
+\left[N_{A}\left(t^{\prime}\right) Z_{A}\left(t^{\prime}\right)\right]\left\{\bar{\delta}_{o}+\bar{\gamma}_{o} /\left(1+\bar{\gamma}_{o} t^{\prime}\right)\right\}>0 .
\end{gathered}
$$

for the daily number of new CoVID-19 cases, providing a self-consistent analytic function for $\rho_{A}\left(t^{\prime}\right)$, instead using $\rho_{A}\left(t^{\prime}\right) \approx \Delta N_{A}\left(t^{\prime}\right) / \Delta t^{\prime}$ as a numerical approximation. For long times, Eq. [4.6] becomes:

$$
\rho_{A}\left(t^{\prime}\right) \approx \mathbf{1}\left\{\bar{\delta}_{o} \bar{G}_{o} \exp \left[+\bar{G}_{o}\right]\right\}\left\{\left[1 /\left(1+\bar{\gamma}_{o} t^{\prime}\right)\right] \exp \left(-\bar{\delta}_{o} t^{\prime}\right)\right\}>0
$$

which exhibits a nearly exponentially decaying tail. Minimizing the rms error using a Logarithmic Y-axis vs linear-time axis gives Figs. 5-6, with these best fit parameter values and results: 


$$
\begin{aligned}
& \log \quad \operatorname{EIM}\left(\bar{\delta}_{o} \neq 0\right) \quad \text { Date or \# Cases or } \\
& Y \text {-axis Value Parameter Value } \\
& \begin{array}{llll}
t^{\prime}=0 & 0.0 \text { days } & 5 / 30 / 2020 & \bar{N}_{00} \approx 0.477
\end{array} \\
& \text { data } \bar{t}_{I}=\quad 13.157 \text { days } \quad 6 / 13 / 2020 \quad \bar{N}_{I}=15,650 \\
& \text { data } \bar{t}_{F}=\quad 88.157 \text { days } \quad 8 / 27 / 2020 \quad \bar{N}_{F}=2,912,774 \\
& \text { calc. } \bar{t}_{\text {Peak }}=\quad 57.157 \text { days } \quad 7 / 28 / 2020 \quad\left(\begin{array}{l}
\bar{\rho}_{o}\left[\bar{t}_{\text {Peak }}\right] \approx \\
\mathbf{5 2 , 3 0 1} / \text { day }
\end{array}\right) \\
& \left(N_{A}^{\max }\right)=\quad---\quad \text { Max.\# cases } \quad 6,621,180 \\
& \bar{K}_{o}=\quad 0.24152 / \text { day } \quad \bar{K}_{A}=\quad 1.778635 / \text { day } \\
& \bar{\alpha}_{S}=\quad 0.0510 / \text { day } \quad \bar{\gamma}_{o}=0.108151 / \text { day }
\end{aligned}
$$

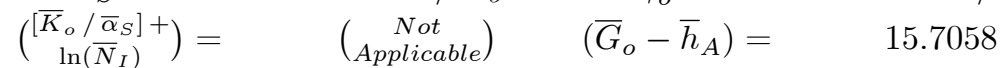

$$
\begin{aligned}
& \bar{h}_{A}=\left|\ln \left(\bar{N}_{00}\right)\right|=\quad 0.74 \quad \bar{\delta}_{o} / \text { day } \quad 0.0081 / \text { day }
\end{aligned}
$$

The Fig. 6 Logarithmic $Y$-axis data fit is quite good, as is Fig. 5 when compared to the IRM Fig. 3. The faster decaying Fig. $5 \rho_{A}\left(t^{\prime}\right)$ tail gives a significantly lower prediction for the total number of Resurgence cases at the pandemic end. Finally, a similar data fit is shown in Figs. 7-8, except the rms error was minimized using a Linear $Y$-axis vs linear-time axis for the $\rho_{A}\left(t^{\prime}\right)$ Resurgence data. It has the following best fit parameters, which are similar to the above Eq. [4.8] table results:

$$
\begin{aligned}
& \begin{array}{cccc}
\text { Linear } & \text { EIM }\left(\bar{\delta}_{o} \neq 0\right) & \text { Date or } & \text { \# Cases or } \\
Y \text {-axis } & \text { Value } & \text { Parameter } & \text { Value }
\end{array} \\
& \begin{array}{cccc}
t^{\prime}=0 & 0.0 \text { days } & 5 / 31 / 2020 & \bar{N}_{00} \approx 0.427
\end{array} \\
& \text { data } \bar{t}_{I}=\quad 12.060 \text { days } \quad 6 / 13 / 2020 \quad \bar{N}_{I}=15,650 \\
& \text { data } \bar{t}_{F}=\quad 87.060 \text { days } \quad 8 / 27 / 2020 \quad \bar{N}_{F}=2,912,774 \\
& \text { calc. } \bar{t}_{\text {Peak }}=\quad 49.060 \text { days } \quad 7 / 20 / 2020 \quad\left(\begin{array}{l}
\bar{\rho}_{o}\left[\bar{t}_{\text {Peak }}\right] \approx \\
\mathbf{5 0}, \mathbf{3 0 0} / \text { day }
\end{array}\right) \\
& \left(N_{\frac{A}{K}}^{\max }\right)=\quad---\quad \text { Max.\# cases } \quad 5,143,380 \\
& \bar{K}_{o}=\quad 0.24067 \quad \bar{K}_{A}=1.96486 / \text { day } \\
& \bar{\alpha}_{S}=\quad 0.0580 \quad \bar{\gamma}_{o}=0.12052 / \text { day }
\end{aligned}
$$

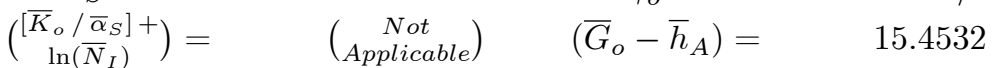

$$
\begin{aligned}
& \bar{h}_{A}=\left|\ln \left(\bar{N}_{00}\right)\right|=\quad \begin{array}{ccc}
\ln \left(\bar{N}_{I}\right) \\
\text { Applicable }
\end{array} \quad \bar{\delta}_{o}=\quad 0.0108 / \text { day }
\end{aligned}
$$

These values form our best estimate for USA CoVID-19 Resurgence. Combining these results with the pre-Resurgence data fit of Figs. 1-2, gives Figs. 9-10 for the full USA CoVID-19 evolution, covering the entire 3/21/2020$8 / 27 / 2020$ time frame. This final data fit captures virtually all of the shape nuances in the actual data. The predicted final number of USA CoVID-19 Cases at the pandemic end, from Eq. [2.7] and Eq. [4.9] is:

$N_{\max }^{T O T A L}=N_{\max }^{o}+N_{A}^{\max }=4,499,494+5,143,380=9,642,874 . \quad[4.10]$

For CoVID-19 in the USA, Fig. 11 plots the ratio of the total number of deaths versus total number of cases (\% vs time), based on the bing.com database $^{1}$, which gives ${ }^{2} 2.9325 \%=(169,108) /(5,766,718)$, as of $8 / 27 / 2020$. This value is similar to the IHME $8 / 27 / 2020$ value $^{5}$ of $~ 3.1065 \%$, which is shown as a horizontal line on Fig. 11.

Using the slightly higher IHME mortality rate allows our Fig. 10 predictions 
to be compared with the most recent IHME predictions ${ }^{5}$, as shown in Fig. 12. The IHME predictions include the presumption of a $2^{\text {nd }}$ Resurgence, due to factors ${ }^{6}$ of "seasonality and declining vigilance". Each IHME projection shown in Fig. 12 is also an IHME Model average ${ }^{5}$, with the magnitude of their lower and upper bound deviations (not graphed) being $<2.5 \%$ by $9 / 26 / 2020$, increasing to $<42 \%$ by $1 / 1 / 2021$. The IHME $2^{\text {nd }}$ Resurgence assumptions are evident in the upward $(+)$ curvature in all IHME predictions, as compared to the downward (-) curvature of the present Resurgence model, indicating progress to a CoVID-19 pandemic shut-off, assuming NO $2^{\text {nd }}$ Resurgence occurs.

The causes of a $2^{\text {nd }}$ Resurgence could include a large-scale set of new reopenings, creating another rapid rise in CoVID-19 cases, similar to Fig. 9. A follow-on analysis would be needed for this $2^{\text {nd }}$ Resurgence. The possibility of multiple CoVID-19 waves was highlighted early on by the University of Minnesota CoVID-19 team ${ }^{7-8}$, but each wave was assumed to have minimal overlap. Instead, these results, and the IHME projections (which already includes a $2^{\text {nd }}$ Resurgence), support the idea that USA CoVID-19 evolution is likely to have multiple overlapping waves of Resurgence.

\section{Discussion and Conclusions}

The Initial Resurgence Model (IRM) for USA CoVID-19 Resurgence, given by Eqs. [3.3a]-[3.3d] and Eqs. [3.6a]-[3.6b] has the $\gamma_{o}$ parameter accounting for the effects of society-wide Social Distancing. Our prior work ${ }^{2}$ showed that the effects of implementing society-wide shut-downs changed the CoVID-19 pandemic evolution within days of the start of its implementation. Thus, the size of $\gamma_{o}$ likely reflects the degree to which society-wide large gatherings were eliminated. It is a non-local parameter that is generally not part of the traditional SEIR (Susceptible, Exposed, Infection, and Recovered or Removed) pandemic modeling, which are governed by local differential equations.

Our analysis shows that the USA CoVID-19 Resurgence data decreased faster than the IRM model predictions. A similar situation ${ }^{3-4}$ was seen in the CoVID-19 pandemic evolution in Italy, which was successfully modeled by introducing a second process:

$$
\bar{\rho}_{\text {data }}\left\{\hat{t}^{\prime}\right\} \sim\left[\exp \left(-\bar{\delta}_{o} \hat{t}^{\prime}\right)\right],
$$

which has an exponentially decaying tail. This second process is independent of the gradually changing $t_{d b l}$ doubling time, which gave rise to the IRM $\left\{\bar{K}_{A}, \bar{\gamma}_{o}\right\}$ parameters.

For USA CoVID-19 Resurgence, an Enhanced Resurgence Model (ERM) was developed to include this second process. This ERM essentially replaces the $\bar{N}_{o}\left[t^{\prime}\right]$ and $\bar{Z}_{o}\left[t^{\prime}\right]$ of Eqs. [3.3a]-[3.3b] with:

$$
\begin{aligned}
& Z_{A}[t]=+\left[\bar{G}_{o} /\left(1+\bar{\gamma}_{o} t\right)\right] \exp \left(-\bar{\delta}_{o} t\right), \\
& N_{A}\left(Z_{A}\right)=\left[e^{+\bar{G}_{o}}\right] \exp \left(-Z_{A}[t]\right) .
\end{aligned}
$$

The necessity of using a second process $\left(\delta_{o} \neq 0\right)$ to model the USA CoVID19 Resurgence has a potentially important implication. This $\delta_{o}$ is a second non-local parameter that may not be part of a traditional SEIR (Susceptible, 
Exposed, Infection, and Recovered or Removed) pandemic model. Pre- vs PostResurgence, what else changed? The most likely explanation is that $\delta_{o}$ measures the degree to which people wear and wore masks to mitigate and prevent CoVID19 spread during the Resurgence.

\section{List of Figures}

Figure 1. Initial Model Baseline (IMB): USA CoVID-19 Projections vs data to 6/7/2020. Predicted Number of Daily CoVID-19 Cases has a peak of 30,727 cases/day on $4 / 15$; with 4,499,494 cases total; and $\sim 5,783$ new cases/day at Day 200 on $9 / 27 / 2020$.

Figure 2. Initial Model Baseline (IMB): USA CoVID-19 Projections vs data to $6 / 7 / 2020$. Revised bing.com data, circa $5 / 3 / 2020$, changed all values back to the pandemic start. Initial Model appears to be a good data fit.

Figure 3. Initial Model fit for CoVID-19 Resurgence: USA Data 6/13/20 to $8 / 8 / 20$. The Fig. $1 I M B$ has data through $6 / 7 / 2020$. It was extrapolated to $8 / 27 / 2020$, then subtracted from the actual data to create Resurgence Only data, which was then fitted using the Initial Model.

Figure 4. USA CoVID-19 Resurgence Only: Data vs Initial Model Fit, 6/13/20 to 8/8/20. Initial Model Baseline (IMB) was subtracted from actual data to set Resurgence Only Data. Resurgence Start Day \#1 was set to 6/13/2020 with $N=15,650$ cases above $I M B$.

Figure 5. Enhanced Initial Model (EIM): USA CoVID-19 Resurgence 6/7/20-8/27/20. EIM best fit with $N_{A}[t]^{\sim} \exp \left(-Z_{A}[t]\right)$ using enhanced $Z_{A}[t]$ function with exponential decay term, which significantly improves fit. Best fit uses Logarithmic Y-axis: $\ln \left(N_{A}[t]\right)$ vs time.

Figure 6. Enhanced Initial Model (EIM): USA CoVID-19 Resurgence 6/7/20-8/27/20. EIM best fit with $N_{A}[t]^{\sim} \exp \left(-Z_{A}[t]\right)$ using enhanced $Z_{A}[t]$ function with exponential decay term. Datafit minimizes rms error on Logarithmic $Y$-axis.

Figure 7. Enhanced Initial Model (EIM): USA CoVID-19 Resurgence 6/7/20-8/27/20. EIM best fit with $N_{A}[t]^{\sim} \exp \left(-Z_{A}[t]\right)$ using enhanced $Z_{A}[t]$ function with exponential decay term. Bestfit minimizes error on Linear $Y$-axis vs time w/ $\mathbf{Y}=d N_{A}[t] / d t=$ Daily \# New CoVID-19 cases.

Figure 8. Enhanced Initial Model (EIM): USA CoVID-19 Resurgence 6/7/20-8/27/20. EIM best fit with $N_{A}[t]^{\sim} \exp \left(-Z_{A}[t]\right)$ using enhanced $Z_{A}[t]$ function with exponential decay term. Deviations on Logarithmic $Y$-axis due to minimizing error using Linear $Y$-axis as given in Fig. 7.

Figure 9. USA CoVID-19 Totals: IMB Plus EIM for Resurgence $3 / 21 / 20$ 8/27/20. Combination of Initial Model Baseline (IMB), starting from 3/21/20 [Fig. 1]; plus Enhanced Initial Model (EIM) for CoVID-19 Resurgence, starting from 6/7/2020 [Fig.7].

Figure 10. USA CoVID-19 Totals: IMB Plus EIM for Resurgence $3 / 21 / 20$ 8/27/20. Combination of Initial Model Baseline (IMB) from 3/21/20 [Fig. 2]; 
plus Enhanced Initial Model (EIM) for CoVID-19 Resurgence from $~ 6 / 7 / 2020$ [Fig. 8] gives $9,642,874$ total at pandemic end.

Figure 11. Net Percent USA CoVID-19 Deaths: Ratio of Total \# of USA Deaths to Total \# of bing.com reported USA CoVID-19 Cases, 3/21/20 through 8/27/2020. Some USA CoVID-19 restrictions lifted 6/7/2020 leading to CoVID Resurgence; IHME used $3.1065 \%$ as of $8 / 27 / 20$.

Figure 12. Net Predicted USA CoVID-19 Deaths: The 8/27/20 IHME $3.1065 \%$ Mortality Rate for USA CoVID-19 cases was applied to Fig. 10 to estimate USA CoVID-19 mortality, assuming NO 2nd Resurgence; enabling comparison to IHME's model, which has 2nd Resurgence effects.

\section{References}

1. www.bing.com/covid: 'Bing COVID-19 Tracker', and https://www.bing.com/covid?form=CPVD07.

2. https://www.MedRxiv.org/content/10.1101/2020.05.04.20091207v1, https://doi.org/10.1101/2020.05.04.20091207, "Initial Model for the Impact of Social Distancing on CoVID-19 Spread", Genghmun Eng.

3. https://www.MedRxiv.org/content/10.1101/2020.06.30.20143149v1, https://doi.org/10.1101/2020.06.30.20143149, "Orthogonal Functions for Evaluating Social Distancing Impact on Covid-19 Spread", G. Eng.

4. https://www.MedRxiv.org/content/10.1101/2020.08.07.20169904v1, https://doi.org/10.1101/2020.08.07.20169904, "Model to Describe Fast Shutoff of CoVID-19 Pandemic Spread", Genghmun Eng.

5. https://covid19.healthdata.org/united-states-of-america?view= total-deaths\&itab=trend, Institute of Health Metrics and Evaluation (IHME), University of Washington, 9/3/2020.

6. https://www.reuters.com/article/us-health-coronavirus-usa/ u-s-coronavirus-deaths-projected-to-more-than-double-to-410000by-january-idUSKBN25V2A7, "U.S. Coronavirus Deaths Projected to More Than Double to 410,000 by January", Daniel Trotta; additional reporting by P. Szekely; editing by H. Goller, 9/4/2020

7. K. A. Moore, M. Lipsitch, J. M. Barry, M. T. Osterholm, "Future of the COVID-19 Pandemic: Lessons Learned from Pandemic Influenza". Center for Infectious Disease and Research [CIDRAP], Academic Health Center, University of Minnesota, April 30, 2020, pp. 1-9.

8. https://www.yahoo.com/news/coronavirus-may-last-2-years-172805061 .html, "Coronavirus May Last 2 Years, Study Warns. Its 2nd wave could be Worse", Dennis Wagner, USA Today, 5/1/20, 10:07 PM PDT. 


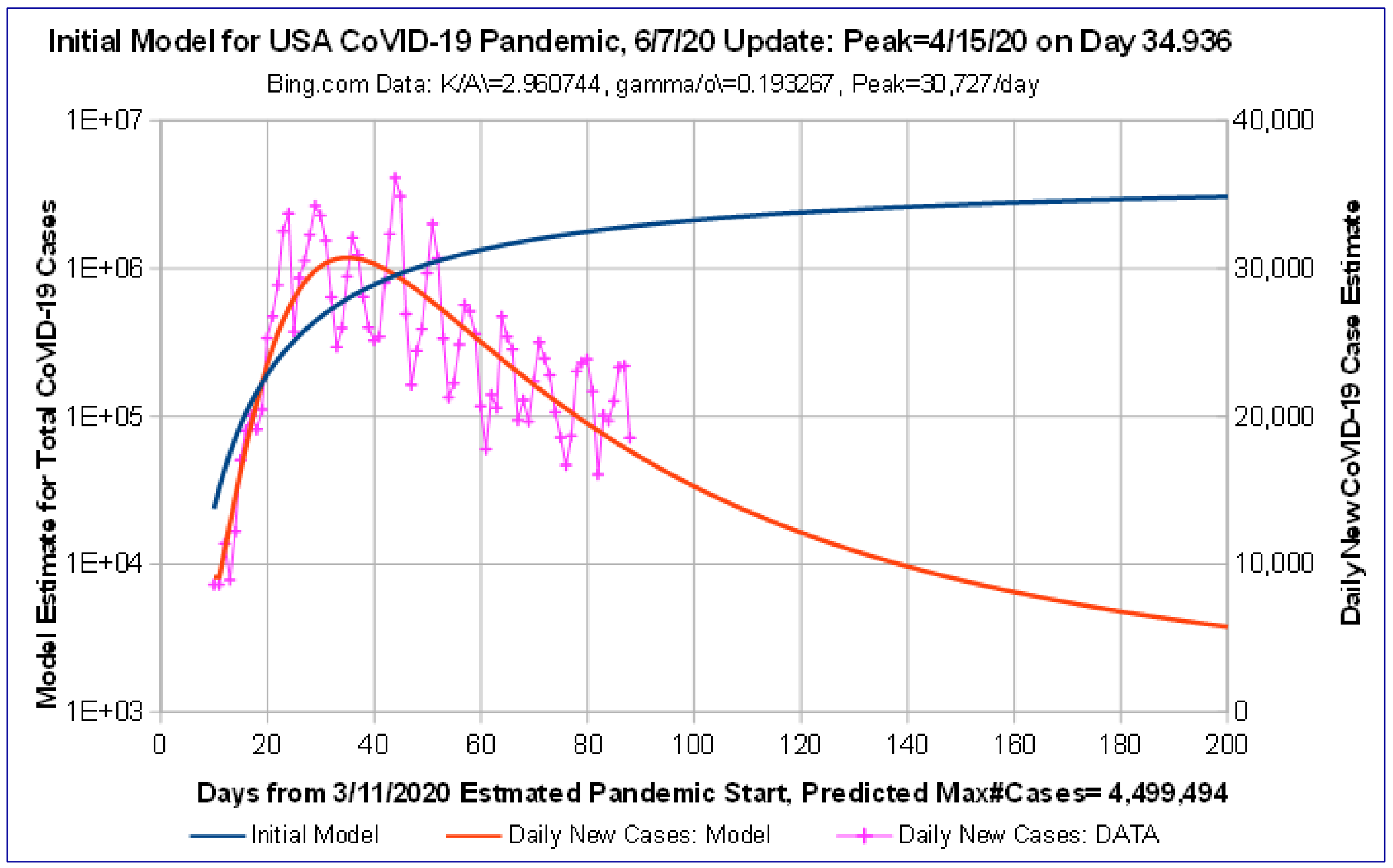

Figure 1. Initial Model Baseline (IMB): USA CoVID-19 Projections vs data to 6/7/2020. Predicted Number of Daily CoVID-19 Cases has a peak of 30,727 cases/day on 4/15; with 4,499,494 cases total; and 5,783 new cases/day at Day 200 on 9/27/2020. 


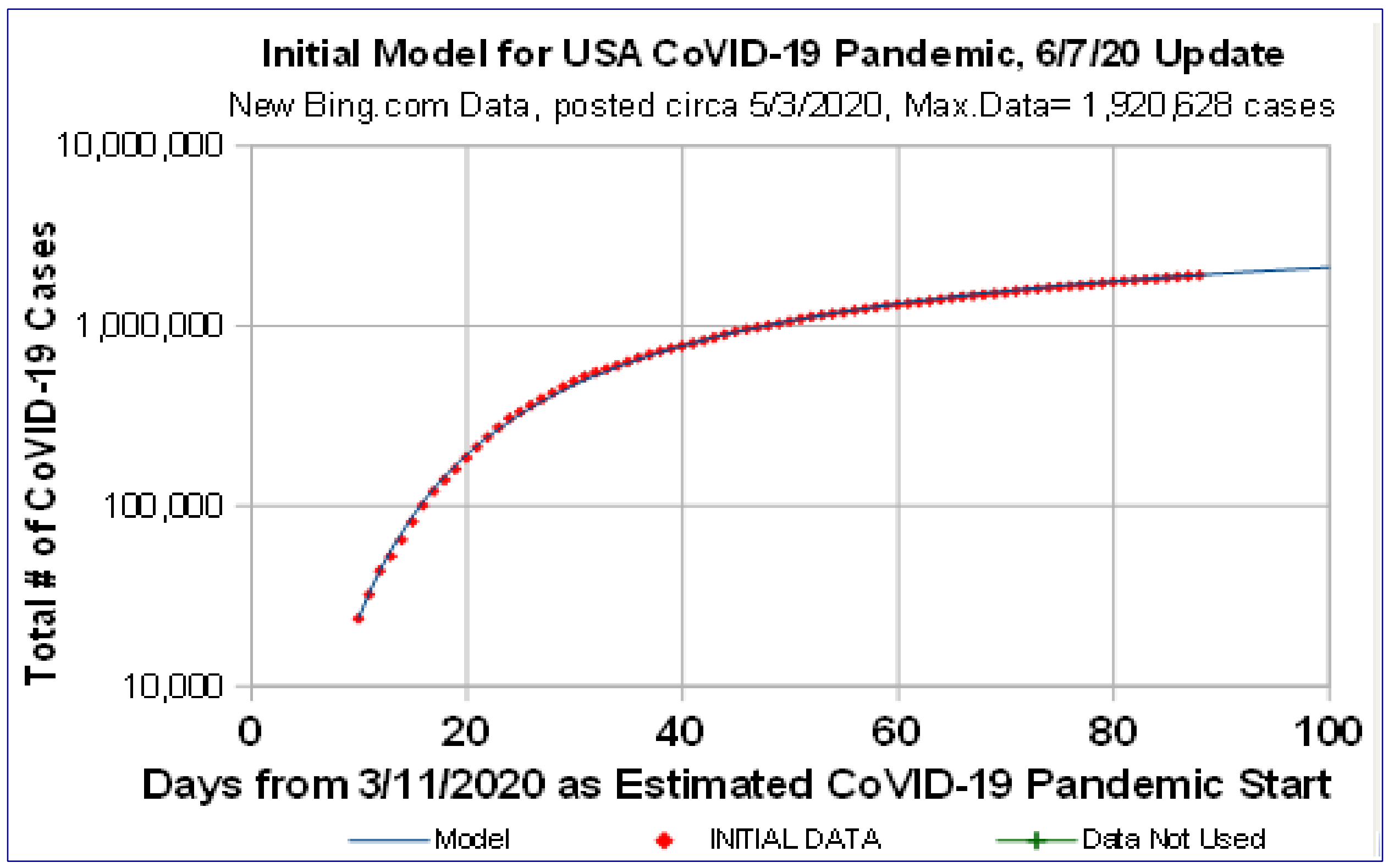

Figure 2. Initial Model Baseline (IMB): USA CoVID-19 Projections vs data to 6/7/2020. Revised bing.com data, circa 5/3/2020, changed all values back to the pandemic start. Initial Model appears to be a good data fit. 


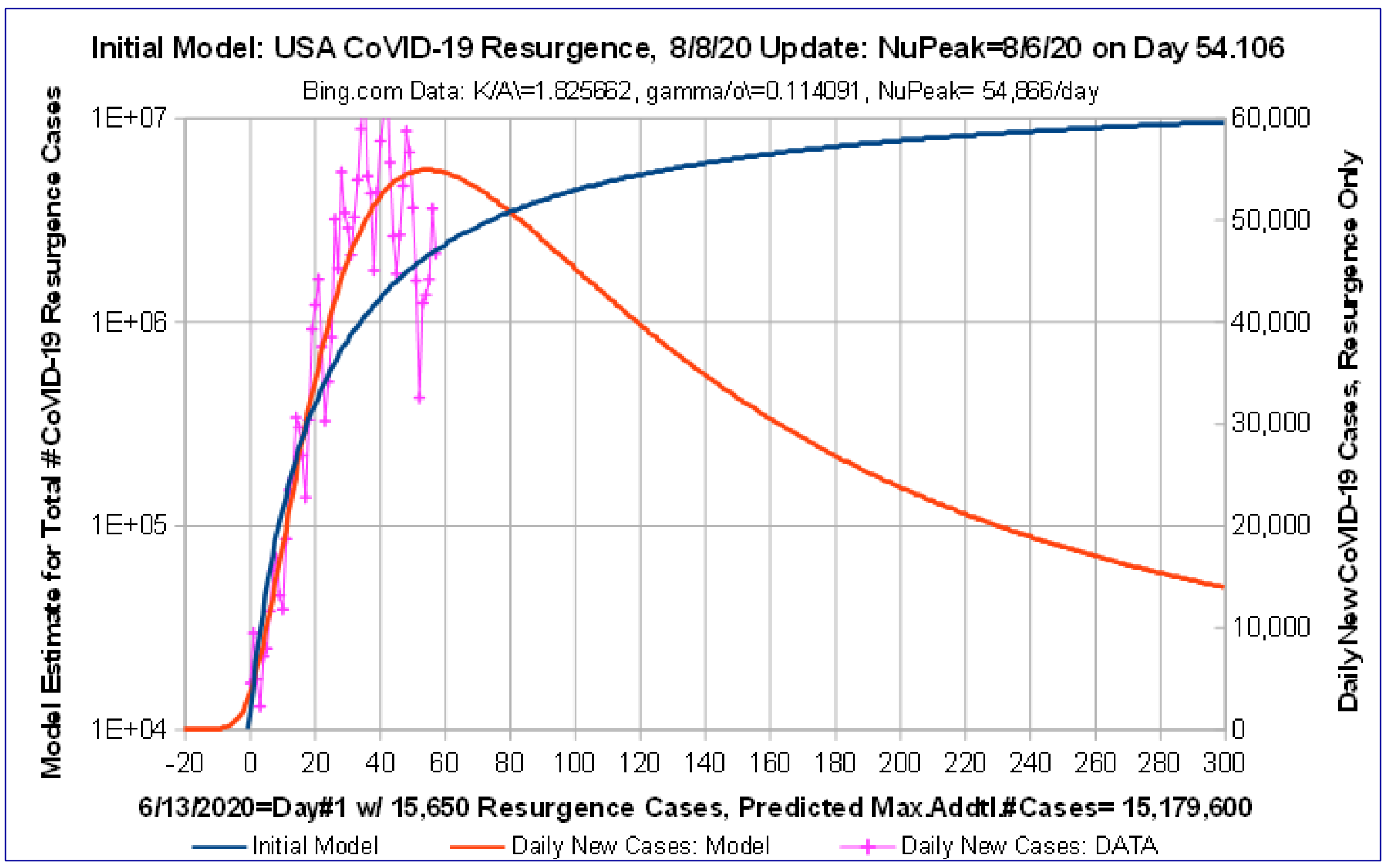

Figure 3. Initial Model fit for CoVID-19 Resurgence: USA Data 6/13/20 to 8/8/20. The Fig. 1 IMB has data through $6 / 7 / 2020$. It was extrapolated to $8 / 27 / 2020$, then subtracted from The actual data to create Resurgence Only data, which was then fitted using the Initial Model. 


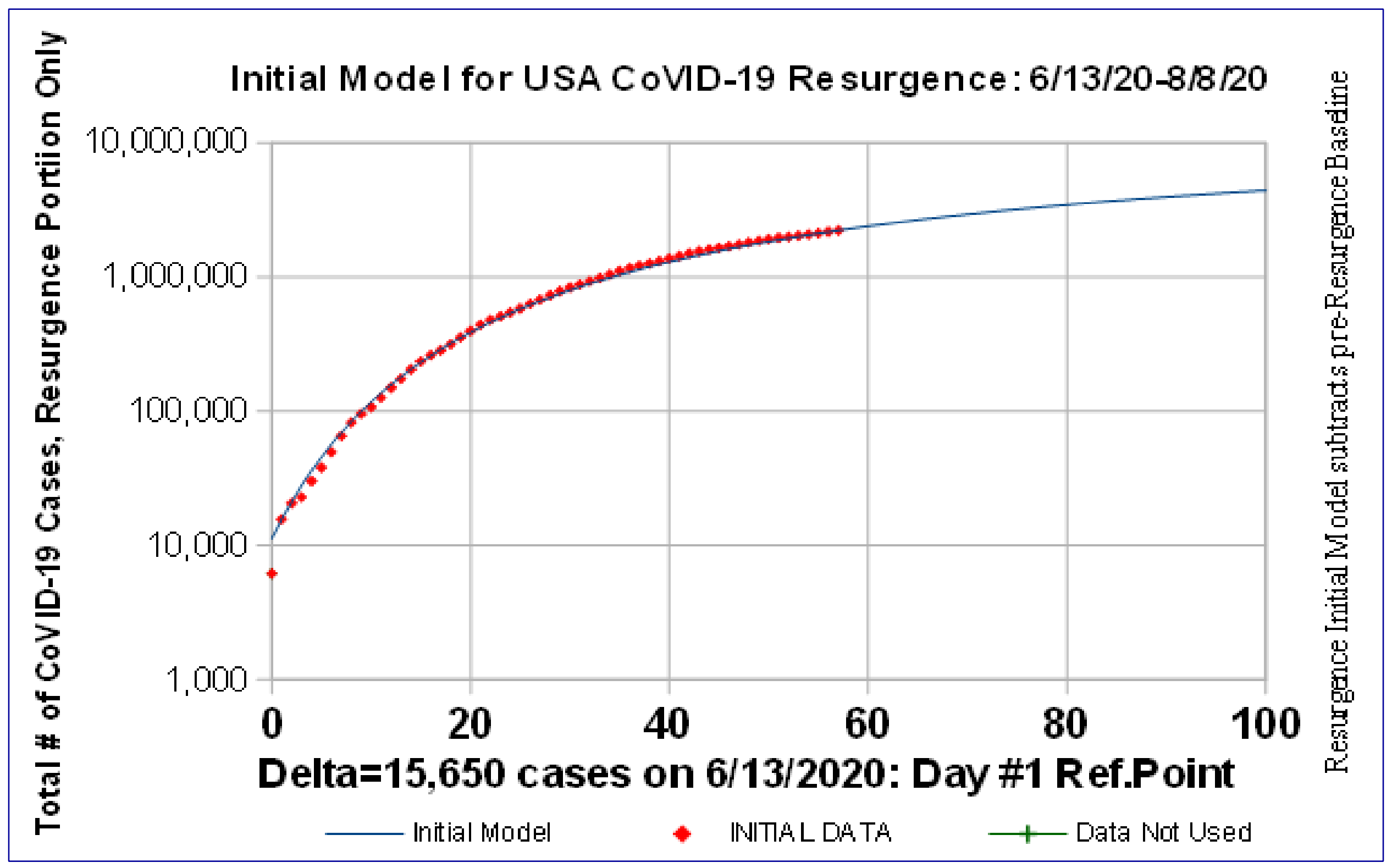

Figure 4. USA CoVID-19 Resurgence Only: Data vs Initial Model Fit, 6/13/20 to 8/8/20. Initial Model Baseline (IMB) was subtracted from actual data to set Resurgence Only Data. Resurgence Start Day \#1 was set to 6/13/2020 with $N=15,650$ cases above IMB. 


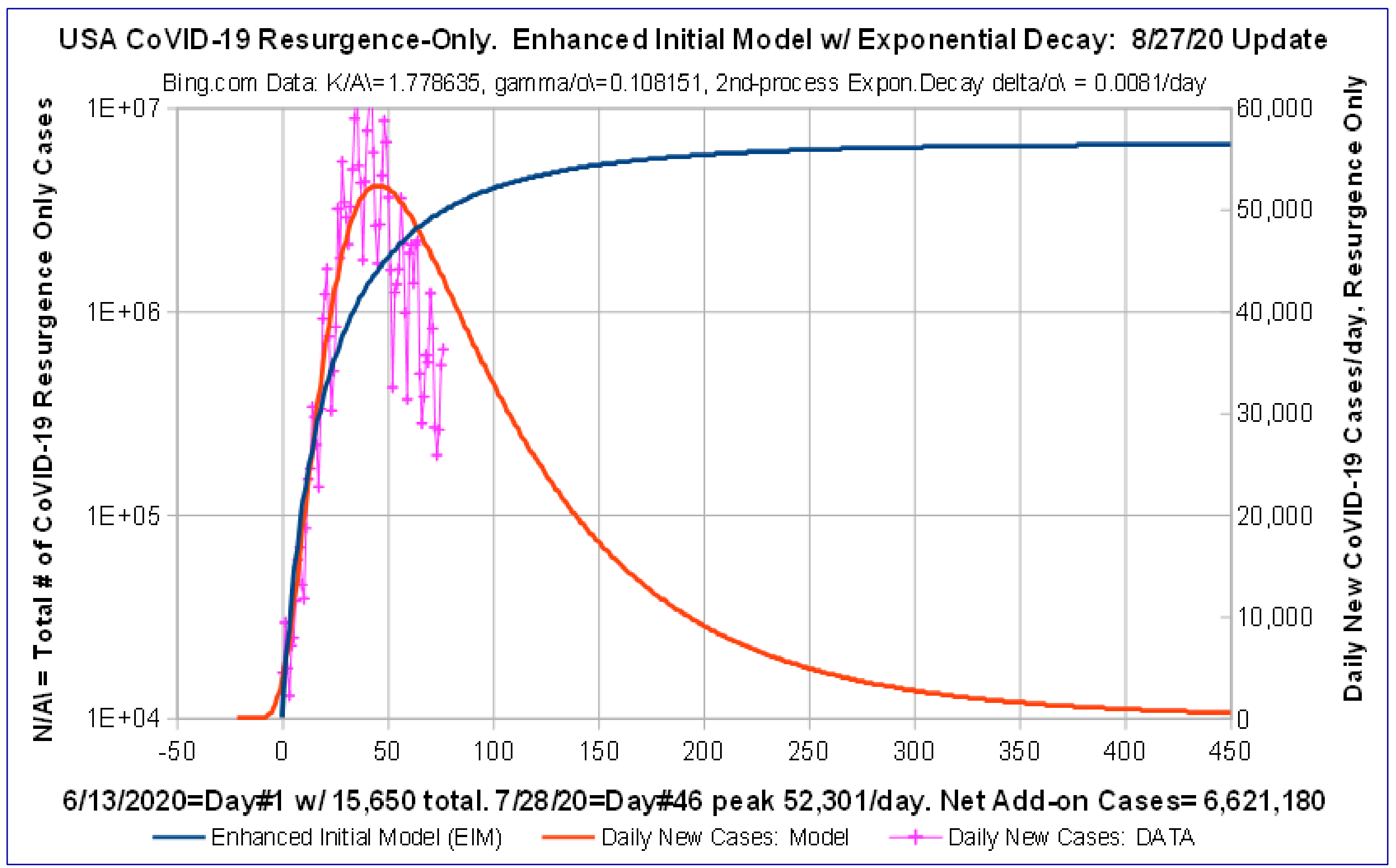

Figure 5. Enhanced Initial Model (EIM): USA CoVID-19 Resurgence 6/7/20-8/27/20. EIM best fit with $N_{A}[t] \sim \exp \left(-Z_{A}[t]\right)$ using enhanced $Z_{A}[t]$ function with exponential decay term, which significantly improves fit. Best fit uses Logarithmic Y-axis: In(NA[t]) vs time. 


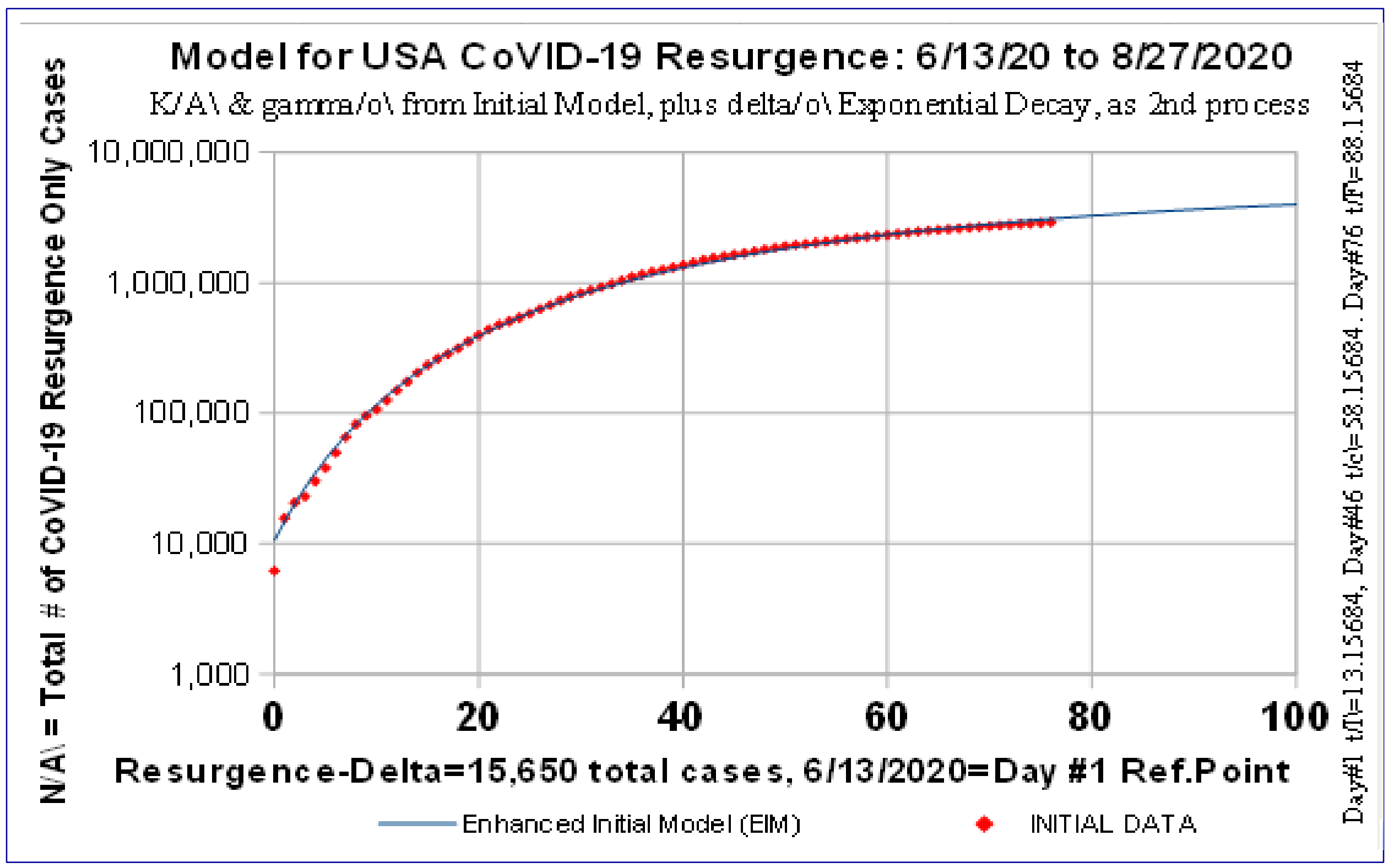

Figure 6. Enhanced Initial Model (EIM): USA CoVID-19 Resurgence 6/7/20-8/27/20. EIM best fit with $N_{A}[t] \sim \exp \left(-Z_{A}[t]\right)$ using enhanced $Z_{A}[t]$ function with exponential decay term. Datafit minimizes rms error on Logarithmic Y-axis. 


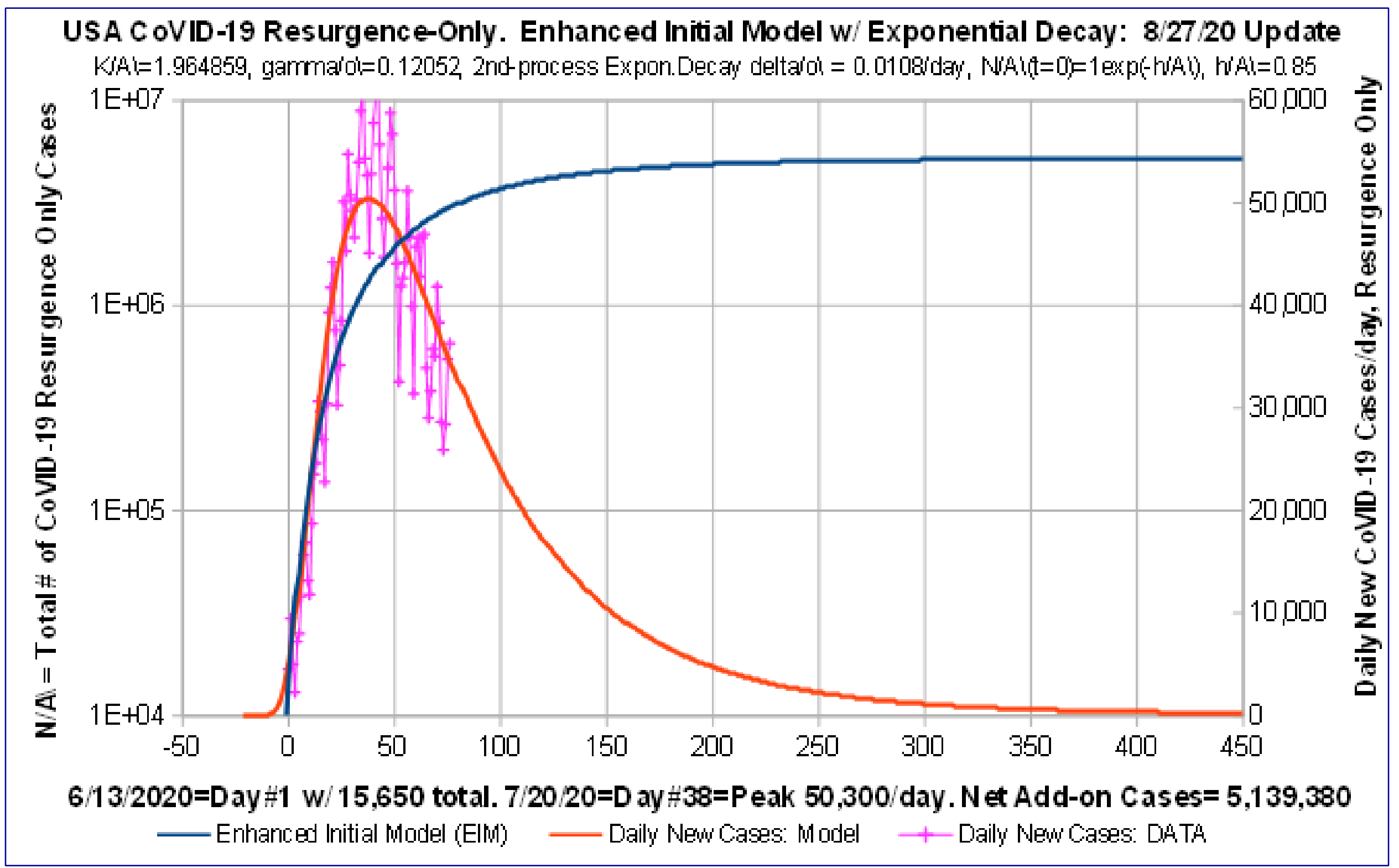

Figure 7. Enhanced Initial Model (EIM): USA CoVID-19 Resurgence 6/7/20-8/27/20. EIM best fit with $N_{A}[t] \sim \exp \left(-Z_{A}[t]\right)$ using enhanced $Z_{A}[t]$ function with exponential decay term. Bestfit minimizes error on Linear $Y$-axis vs time $w / Y=d N A[t] / d t=$ Daily \# New CoVID-19 cases. 


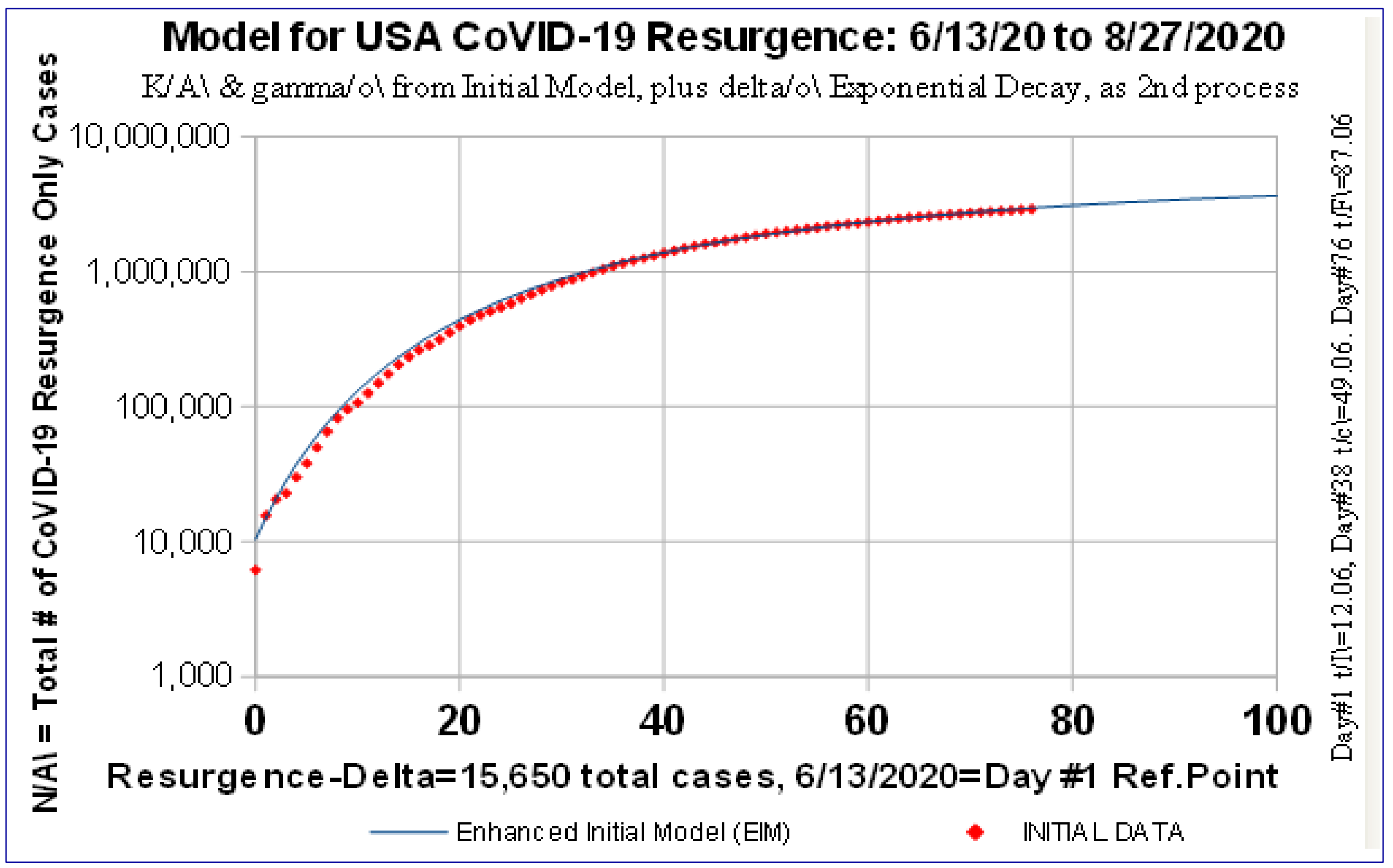

Figure 8. Enhanced Initial Model (EIM): USA CoVID-19 Resurgence 6/7/20-8/27/20. EIM best fit with $N_{A}[t] \sim \exp \left(-Z_{A}[t]\right)$ using enhanced $Z_{A}[t]$ function with exponential decay term. Deviations on Logarithmic Y-axis due to minimizing error using Linear Y-axis as given in Fig. 7. 


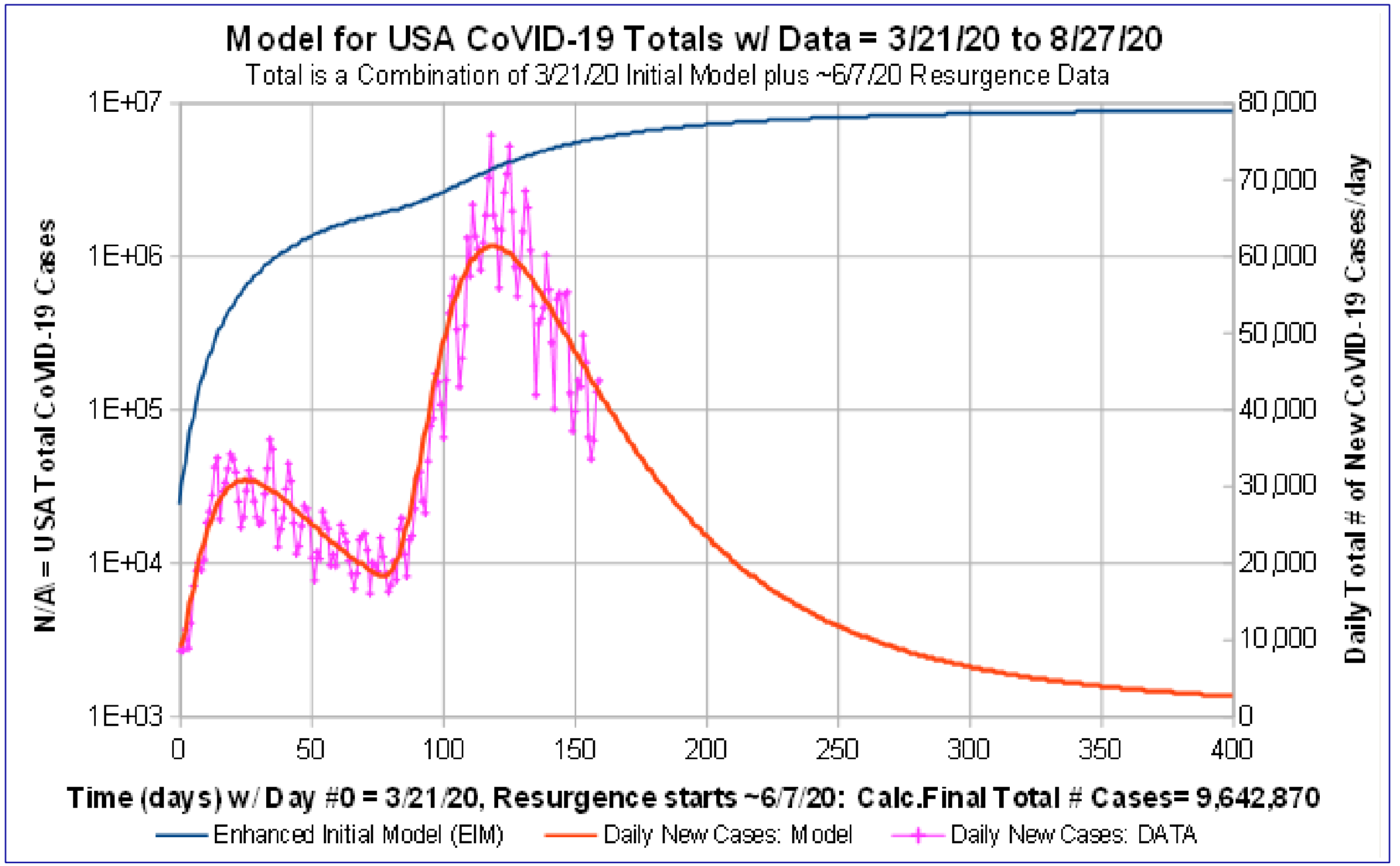

Figure 9. USA CoVID-19 Totals: IMB Plus EIM for Resurgence 3/21/20-8/27/20. Combination of Initial Model Baseline (IMB), starting from 3/21/20 [Fig. 1]; plus Enhanced Initial Model (EIM) for CoVID-19 Resurgence, starting from 6/7/2020 [Fig.7]. 


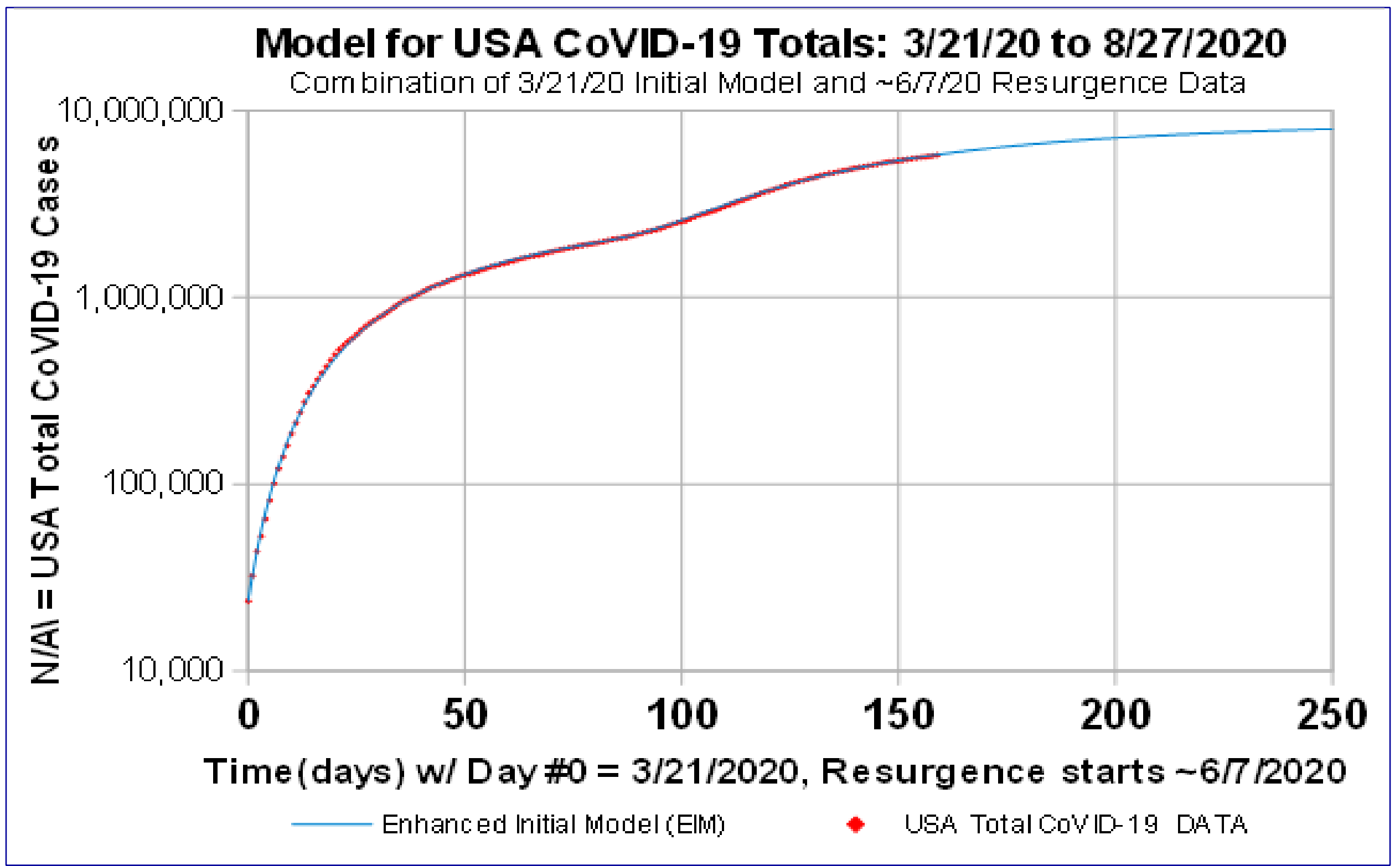

Figure 10. USA CoVID-19 Totals: IMB Plus EIM for Resurgence 3/21/20-8/27/20. Combination of Initial Model Baseline (IMB) from 3/21/20 [Fig. 2]; plus Enhanced Initial Model (EIM) for CoVID-19 Resurgence from 6/7/2020 [Fig. 8] gives 9,642,874 total at pandemic end. 


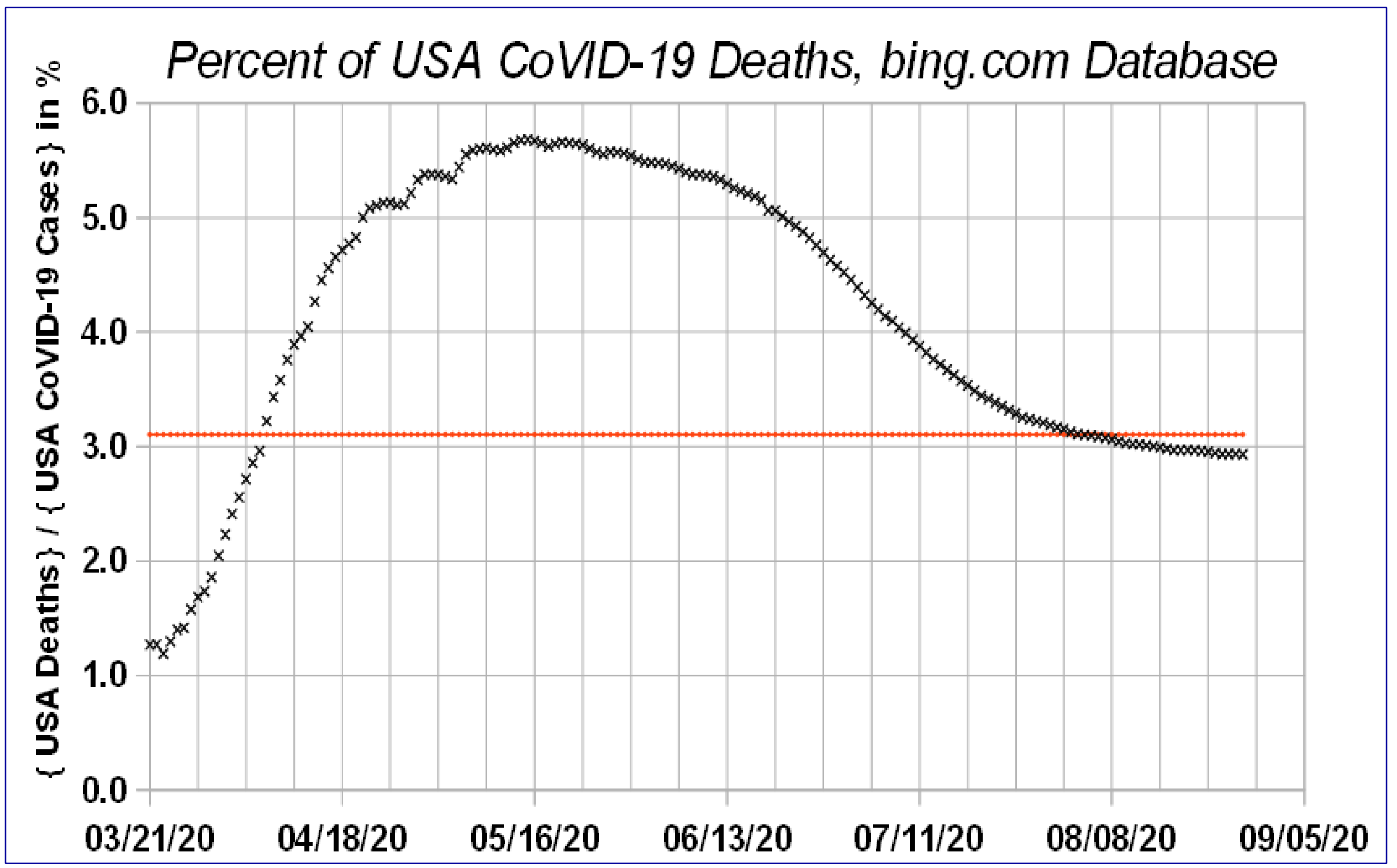

Figure 11. Net Percent USA CoVID-19 Deaths: Ratio of Total \# of USA Deaths to Total \# of bing.com reported USA CoVID-19 Cases, 3/21/20 through 8/27/2020. Some USA CoVID-19 restrictions lifted $\sim 6 / 7 / 2020$ leading to CoVID Resurgence; IHME used $3.1065 \%$ as of 8/27/20. 


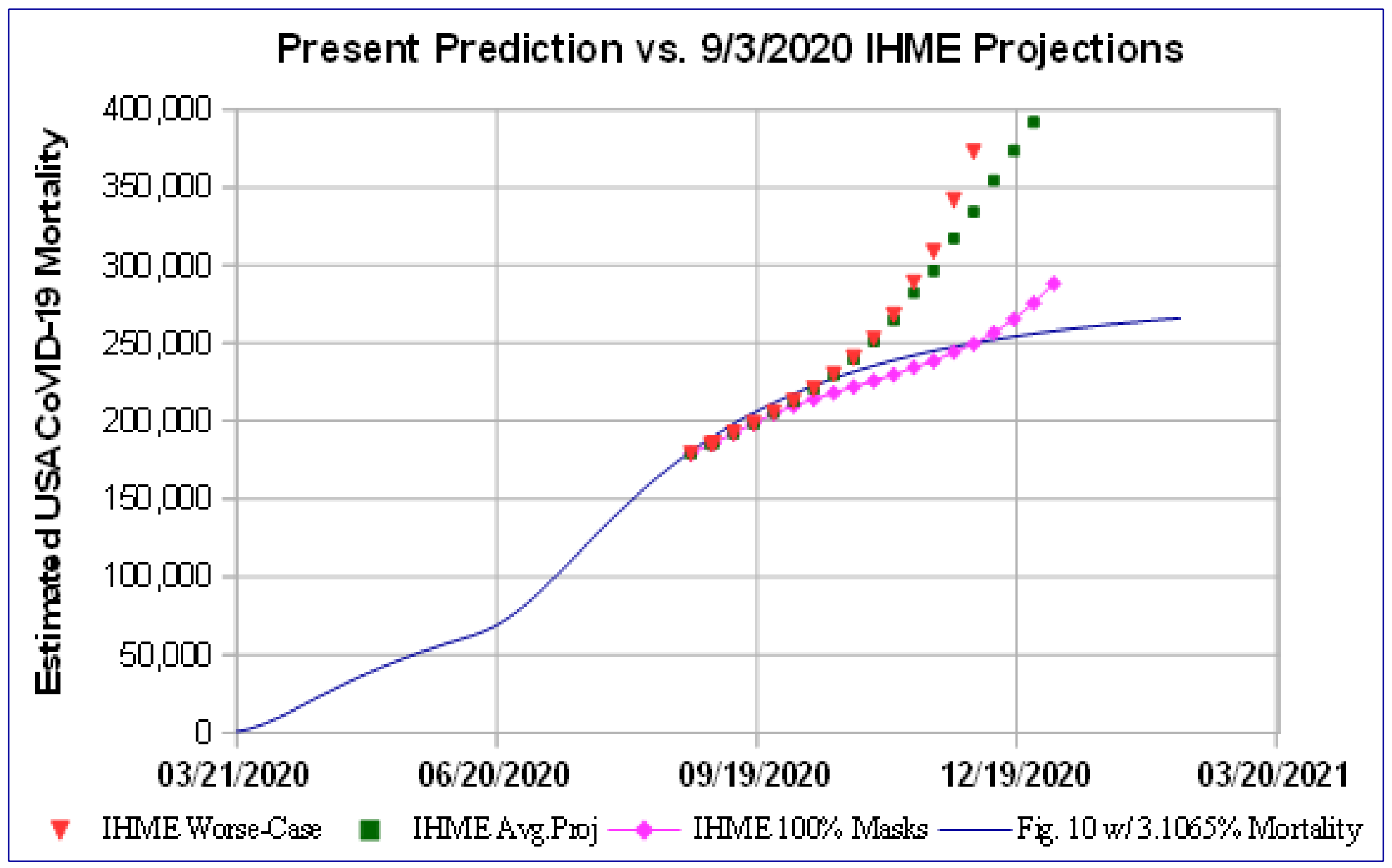

Figure 12. Net Predicted USA CoVID-19 Deaths: The 8/27/20 IHME 3.1065\% Mortality Rate for USA CoVID-19 cases was applied to Fig. 10 to estimate USA CoVID-19 mortality, assuming NO 2nd Resurgence; enabling comparison to IHME's model, which has 2nd Resurgence effects. 